\title{
RHIC BEAM LOSS MONITOR SYSTEM INITIAL OPERATION*
}

\author{
R. L. Witkover ${ }^{\ddagger}$, R. J. Michnoff and J. M. Geller \\ Brookhaven National Laboratory \\ Upton, NY, USA 11973
}

\begin{abstract}
The RHIC Beam Loss Monitor (BLM) System is designed to prevent beam loss quenching of the superconducting magnets, and acquire loss data. Four hundred ion chambers are located around the rings to detect losses. The required 8-decade range in signal current is compressed using an RC pre-integrator ahead of a low current amplifier. A beam abort may be triggered if fast or slow losses exceed programmable threshold levels. A micro-controller based VME module sets references and gains and reads trip status for up to 64 channels. Results obtained with the detectors in the RHIC Sextant Test and the prototype electronics in the AGS-to-RHIC (AtR) transfer line are presented along with the present status of the system.
\end{abstract}

\section{THE DETECTORS AND ELECTRONICS}

It has been estimated that the RHIC superconducting magnets will quench for a fast (single turn) loss $>2 \mathrm{~mJ} / \mathrm{g}$ or a slow $(100 \mathrm{msec})$ loss $>8 \mathrm{~mW} / \mathrm{g}$. These correspond to a signal current range from $5.5 \mathrm{~mA}$ for fast loss at injection, and $17.6 \mathrm{nA}$ for a slow loss at full energy. Allowing for studies, a dynamic range of 8 decades in detector current is required. The processed BLM signal is digitized at $720 \mathrm{~Hz}$ and continually compared to programmable fast and slow loss abort levels. An abort halts data acquisition providing a 10 second loss history. BLM parameters may be adjusted to set gains, fast and slow loss thresholds and abort mask bits on specific RHIC Event Codes.

The RHIC Ring BLM system uses 400 FNAL Tevatron type ion chambers [1] modified by using an isolated BNC to break the ground loop formed by the signal and HV cable shields. Rexolite rather than PTFE is used for the insulators in the BNC and SHV connectors to improve the radiation hardness. The ion chamber[2] consists of a 113 cc glass bulb filled with argon to about 725 milli-Torr. Each chamber is calibrated using a cesium-137 source. The mean sensitivity in the middle of the plateau ( 1450 Volts) is $19.6 \mathrm{pA} / \mathrm{rad} / \mathrm{h}$, with $95 \%$ within $\pm 1.5 \mathrm{pA} / \mathrm{rad} / \mathrm{hr}$ of the mean. The high voltage is normally set to $1450 \mathrm{~V}$ with a $3000 \mathrm{~V}$ upper limit. Half of the ion chambers (198) are mounted on the quadrupole cryostats between the Rings using stainless steel "belly bands". Ninety-six

*Work supported by the US Department of Energy

"witkover@bnl.gov
BLMs are placed at insertion region quads. In the warm regions, 68 detectors are mounted on the beam pipe at sensitive loss points. In addition, $38 \mathrm{BLMs}$ are available as re-locatable monitors.

Belden 9054, a low noise, non-tribo-electric RG-59 equivalent cable was used for signal transmission. The HV bias voltage is carried on red RG-59 cable which is daisy-chained from detector to detector. Two HV cables are used in each sector, picking up alternate BLMs to provide some redundancy in the event of a high voltage short. An RC network built into each ion chamber housing provides decoupling in the case of a short, noise filtering, and storage capability to handle large fast losses.

The analog electronics are packaged in VME modules, each of which interfaces to 8 BLMs. Up to 8 analog boards are managed by a micro-controller module which is independent of the crate front-end computer (FEC) once the event-driven write list values have been set through high level code. This insulates the real-time operations from the control system $\mathrm{I} / \mathrm{O}$, allowing the BLM system to operate during an FEC link failure. Commercial digital I/O and DAC modules control the HV power supplies. The electronics are located in service buildings at 2,4,5,7,8,10 and 12 oclock, allowing access during beam storage. Standard VME crates were modified for the special needs of the BLM electronics. Tests indicated that the standard \pm 12 Volt switcher power supplies were too noisy for the high sensitivity analog circuitry. On-board DC-DC converters were not used due to limited real estate and the possibility of oscillator noise. It was decided to incorporate a separate linear \pm 15 Volts supply into the crate to power the analog modules. A piggy-back board across the last nine $\mathrm{P} 2$ connectors provides a dedicated bus between the micro-controller module and the 8 analog modules.

\subsection{The Analog Module}

Figure 1 shows a simplified schematic of one channel of the Analog Module. The input RC filter matches the magnet thermal time constant and integrates any fast loss, greatly reducing the dynamic range required, while providing a sufficient signal to protect against a fast loss quench. Back-biased matched low leakage diodes (DPAD-5) protect the amplifier input from high voltage spikes. The OPA627AU low current amplifier is rolled 
off to a $10 \mu$ sec rise time. To allow for BLM shielding differences, jumpers can set three alternate gains.

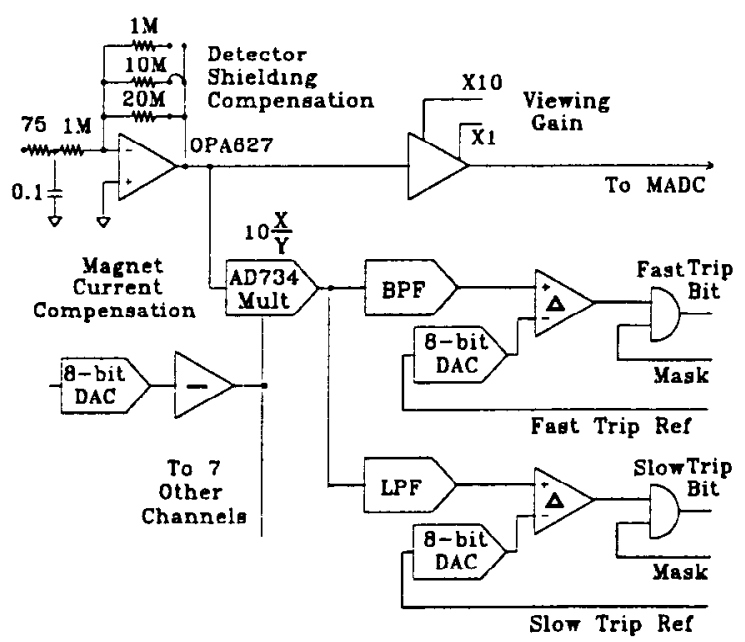

Figure 1. Schematic of a typical channel of the BLM Analog Module.

A second amplifier provides programmable gains of 1 or 10 in the signal acquisition path. The data is read at 720 $\mathrm{Hz}$ by a RHIC VME MADC [3] configured for $\pm 10 \mathrm{~V}$, 13-bits, and stored in a 2 Mbyte on-board memory. An optional off-board $360 \mathrm{~Hz}$ anti-aliasing filter is available. Readings can be taken at additional times as required for specific applications. For the nominal jumper setting and a buffer gain of 10 , one LSB represents $12.5 \mathrm{pA}$, comparable to the noise observed in beam tests. Offsets, typically a few LSBs at unity viewing gain, are not adjustable since these can be removed in the higher level processing.'

The first stage output also goes to an AD734 analog multiplier which provides gain to compensate for the increased magnet quench sensitivity with current. An 8-bit DAC sets the gain for all multipliers on a board. A high pass $(100 \mu \mathrm{sec})$ and low-pass $(20 \mathrm{msec})$ filter direct the signal to respective fast or slow loss comparators with independent programmable references. The gain provided by the multiplier cuts the dynamic range so 8-bit reference DACs are sufficient. Each comparator may be masked to prevent a bad BLM from inhibiting the beam or to allow special conditions. The gains, mask bits and trip levels may be changed by events on the RHIC Event Link. Any trip latches the state allowing the trip location to be determined. An Altera 7128 chip is programmed to perform all logic and communication functions with the BLM Micro-controller module via the dedicated bus on the VME P2 backplane.

\subsection{The Micro-Controller Module}

The RHIC Control System communicates with the Analog Modules via the BLM Micro-Controller [4].
Once the Micro-Controller receives the Write-list containing the array of set-points (gains, thresholds, masks, etc.) it completely controls the Analog Module, freeing the FEC for other controls tasks and allowing the BLM system to operate even in the event of a controls failure. A Microchip PIC16C64 micro-controller services the 256 byte registers on the BLM Analog modules. A $64 \mathrm{k}$ $x 16$ bit memory holds the Write-lists for 256 RHIC Event codes, each associated with up to 255 address/data values. On detection of a specific Event, the corresponding Write-list is sequentially executed with the data going to a particular register. Altera 9320 and 7128 gate arrays are used on the board.

\section{BLM TEST RESULTS}

Figure 2 shows the responses of the circuitry to 8 bunches of $2 \times 10^{12}$ protons each, extracted at $30 \mathrm{~Hz}$ rate. The top trace is the analog output showing the signal rising rapidly due to the loss from each bunch transfer. It then decays with the $100 \mathrm{msec}$ front-end filter time constant. The middle trace is the slow loss filter output clearly showing rejection of the fast losses. The bottom trace is the comparator output for a 2 Volt reference.

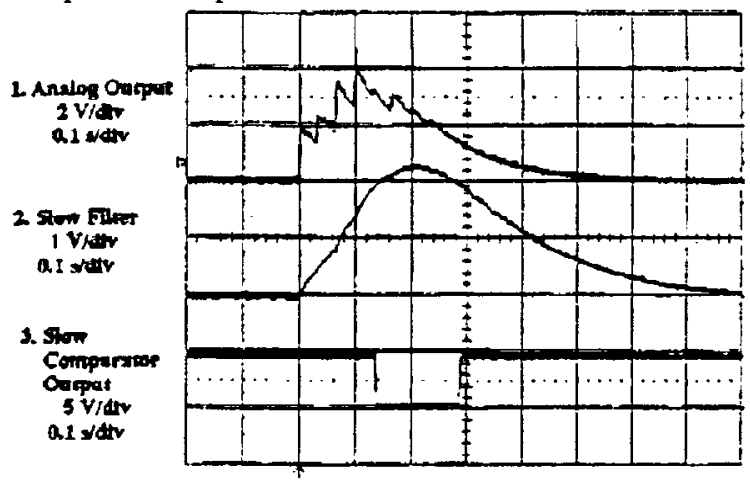

Figure 2. Losses from eight bunch $2 \times 10^{12}$ protons/bunch transfer, Slow Filter and Comparator outputs

The top trace of Figure 3 is another cycle of 8 extracted proton bunches. The middle trace is the fast loss filter output, showing only the fast component of the signal. The

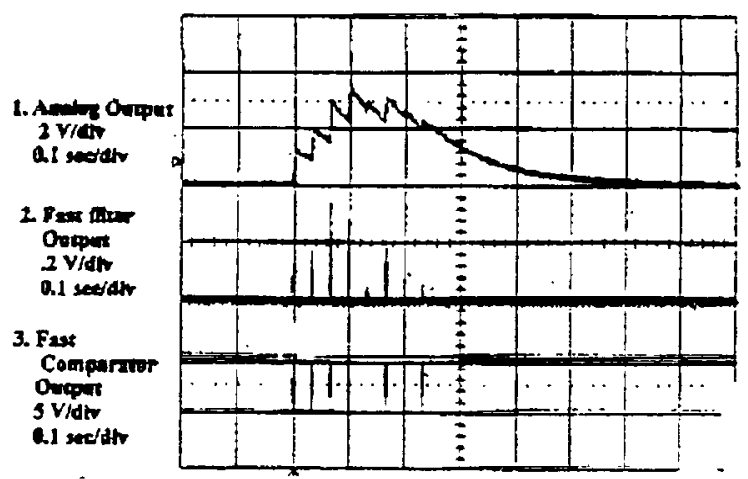

Figure 3. Losses from eight bunch $2 \times 10^{12}$ protons/bunch transfer, Fast Filter and Comparator outputs 
bunches did not have equal losses so the comparator did not trip for every transfer. It is clear that the circuit has the ability to discriminate between fast and slow losses.

In January 1997 single bunches of an $\mathrm{Au}^{+79}$ beam were injected into RHIC Sectors 4 and 5 of one ring of superconducting magnets. In this sextant BLMs were on the inside of the Ring carrying the beam. Since sufficient Ring electronics were not yet available, and the beam was single pass, AtR loss monitor electronics (low-leakage integrators) were used. For normal injection conditions the loss signals were easily observed even though the intensity of $1 \times 10^{8} \mathrm{Au}^{79}$ ions was an order of magnitude below the design value. A large loss was purposely created at $S=600$ meters. The data with the background loss signal subtracted is shown in Figure 4. With the BLMs located at the quads (about 15 meter interval) it can be seen from the data that there is sufficient spatial resolution and dynamic range to determine the direction of the beam causing the loss. The observed integrator noise was of the order of a few LSBs, consistent with results in the AtR line. Noise due to the injection kickers and RF was not noticeably greater.

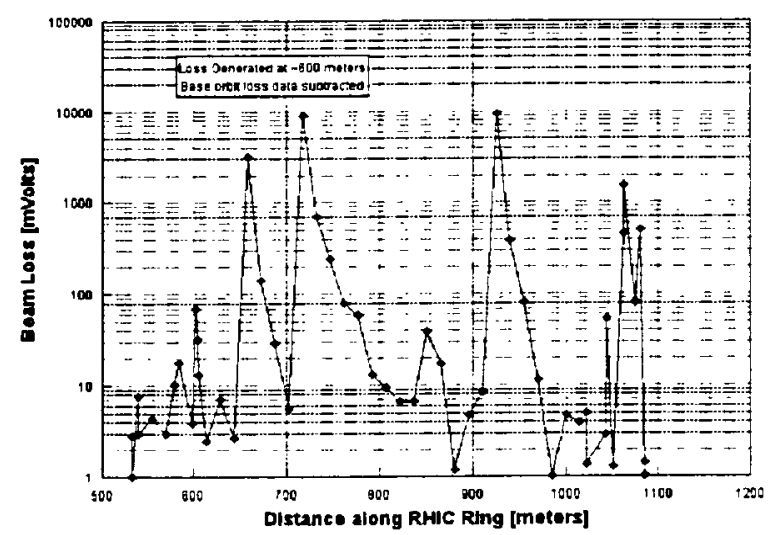

Figure 4. Beam loss along RHIC Sectors 5 and 4 due to test loss at 600 meters for $1 \times 10^{8} \mathrm{Au}^{+79}$ ions, with the background loss signal subtracted

The data shown in Figure 4 covers a 4 decade range and was taken without switching gain. In other runs larger losses were purposely generated, requiring lowering the integrator gain. These results, and the low observed noise, indicted that the BLM system will have sufficient range to meet the design requirements.

\section{SYSTEM STATUS}

All eleven BLM VME crates have been installed in the service buildings around the RHIC tunnel. The crates were accessed through the RHIC Control System to calibrate the electronics. The calibration consisted of injecting signals from a precision current source at several different currents into each channel. The offset and gain data were then entered in the system database. Cabling has been installed, terminated and tested. The system is ready for first beam to be circulated in RHIC.

\section{ACKNOWLEDGEMENTS}

Pat Thompson, as system commissioner, was a key figure in the system check out, siting of detectors and beam testing. He was also responsible for integrating the BLMs into the Contols database. Paul Ziminski was responsible for calibrating the detectors in the lab and, together with Ron Schroeder, the field calibration of the electronics. Much of the work on the electronics design was done by Emil Zitvogel. Barry Clay was responsible for setting up the LabView testing of the Analog Modules.

\section{REFERENCES}

[1] Shafer, R. E., et al., "the Tevatron Beam Position and Beam Loss Monitoring Systems", Proc. The $12^{\pi}$ Int'l. Conf. High Energy Accel., p609, 1983

[2] Troy-lonic Inc., 88 dell Ave, PO Box 494, Kenvil, NJ 07847

[3] Michnoff, R., "The RHIC General Purpose Multiplexed Analog-toDigital Convertor System", Proc 95 Particle Accel. Conf., IEEE Cat. No. $95 \mathrm{CH} 35843, \mathrm{p} 2229,1995$

[4] Michnoff, R., see

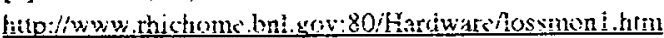

